\title{
Fractionation and Identification of Antioxidant Compounds from Bran of Thai Black Rice $c v$. Riceberry
}

\author{
Panawan Suttiarporn, Phumon Sookwong, and Sugunya Mahatheeranont
}

\begin{abstract}
Bran of Thai Black Rice cv. Riceberry was extracted using solvent extraction (hexane, dichloromethane and methanol). The crude extracts obtained were investigated for their antioxidant property and total phenolic contents which were assessed by 1,1-diphenyl-2-picrylhydrazyl (DPPH) free radical scavenging assay and Folin-Ciocalteu's method, respectively. Results showed that the methanolic extract had the strongest total antioxidant activity and phenolic content at $97.83 \%$ and $9.87 \mathrm{mg} \mathrm{GAE} / \mathrm{ml}$ of extract, respectively. The methanolic extract was further fractionated by column chromatography to obtain ten fractions. The chemical components of the crude extracts and the fractions were analyzed by gas chromatography-mass spectrometry (GC-MS). Seventy-nine constituents were identified in all extracts of Riceberry bran. Among them, 9 simple phenolics and 4 flavonoids, which were previously reported to have antioxidant activities, were quantified. The major simple phenolic was 4-vinylguaiacol and the major flavonoid was apigenin.
\end{abstract}

Index Terms-Riceberry, antioxidants, fractionation, gas chromatography mass-spectrometry.

\section{INTRODUCTION}

Black rice brans, the black outer layers removed during the milling of black rice, have potential to promote human health due to the great antioxidant potency of their phytochemicals. These compounds include anthocyanidins (cyanidin-3-O-glucoside and peonidin-3-O-glucoside [1]), flavonoids glycosides (quercetin-3-O-glucoside, isorhamnetin-3-O-glucoside and myricetin-7-O-glucoside [2]), simple phenolics (2-methoxy-4-vinylphenol (4-vinylguaiacol), 2-methoxy-phenol (guaiacol), and 1,2-benzendiol (catechol) [3]), vitamin E, (tocopherols and tocotrienols [4]), and $\gamma$-oryzanols (ferulate derivatives of 24-methylenecycloartenol, cycloartenol, campesterol and $\beta$-sitosterol [5]).

Riceberry rice, recently developed for nutritional benefit to consumers, a combination of Hom Nin rice, with its well-known antioxidant properties, and Thai Hom Mali rice, which is also called Thai Jasmine or Khao Dawk Mali 105. The extracts of the bran had been reported to have potential anticancer activity [6], hypoglycemic, hypolipidemic, antioxidant, and anti-inflammation properties [7].

There are a few reports about the identification of chemical components in pigmented rice by GC-MS. However, GC-MS

Manuscript received April 19, 2015; revised June 26, 2015. This work was supported by the Nation Research Council of Thailand (NRCT), the Commission on Higher Education (CHE) and Center of Excellence for Innovation in Chemistry (PERCH-CIC), Thailand.

The authors are with Center of Excellence for Innovation in Chemistry and Department of Chemistry, Faculty of Science, Chiang Mai University, Chiang Mai 50200, Thailand (e-mail: Panawansut@gmail.com, phumon@gmail.com, sugunya.m@cmu.ac.th). analysis of some specific chemical components from fractions isolated by column chromatography have not been reported. Therefore, the objective of this research was to isolate complex crude extracts of Riceberry bran, to subfractionate the extract using column chromatography, and identify of antioxidant components by GC-MS.

\section{MATERIALS AND METHODS}

\section{A. Plant Material}

Thai black rice cv. Riceberry was collected from the experimental field in Kasetsart University, Kamphaengsaen Campus, Nakorn Pathom province in the central Thailand.

\section{B. Extraction and Fractionation}

Bran of Riceberry $(1.00 \mathrm{~kg}$ ) was extracted with $2.5 \mathrm{~L}$ of hexane, $2.5 \mathrm{~L}$ of dichrolomethane, and $2.5 \mathrm{~L}$ of methanol, respectively. Then, each extract was evaporated to dryness yielding hexane extract $(\mathrm{CH}, 141.19 \mathrm{~g})$, dichloromethane extract (CDCM, $44.21 \mathrm{~g}$ ) and methanol extracts (CM, 58.66 g). Part of the methanol extract $(30.00 \mathrm{~g})$ was fractionated with siliga gel and eluted by a series of solvents with increasing polarity; hexane, hexane:ethyl acetate, ethyl acetate:methanol and methanol yielding 10 fractions which were in agreement of those on TLC patterns; CM1 (6.01 g), CM2 (1.18 g), CM3 (6.49 g), CM4 (1.42 g), CM5 (0.58 g), CM6 (0.73 g), CM7 (0.36 g), CM8 (6.35 g), CM9 (3.63 g) and CM10 (2.55 g).

\section{1,1-Diphenyl-2-Picrylhydrazyl Free Radical Scavenging Assay}

An aliquot $3 \mathrm{~mL}$ of the DPPH radical solution $(0.3 \mathrm{mM}$, in methanol) was added to $1 \mathrm{mg} / \mathrm{mL}$ of the extract. The mixture was shaken and left to stand for $30 \mathrm{~min}$ at room temperature in the dark. The absorbance was measured at $517 \mathrm{~nm}$. The radical scavenging effect was calculated by the following equation: scavenging activity $(\%)=100 \times[$ (Abs of control Abs of Sample)/ Abs of control].The experiment was performed in triplicate.

\section{Determination of Total Phenolic Contents}

Total phenolics in the extracts were investigated using Folin-Ciocalteu's method. The reaction mixture was prepared by mixing $0.2 \mathrm{ml}$ of methanolic solution of extract, $1 \mathrm{ml}$ of Folin-Ciocalteu's reagent, $1 \mathrm{ml} 7 \% \mathrm{NaHCO}_{3}$ and $5 \mathrm{ml}$ of distilled water. The samples were stand for 1 hour at room temperature. The absorbance was determined at $765 \mathrm{~nm}$. The content of phenolics in extracts was expressed in terms of gallic acid equivalent ( $\mathrm{mg}$ of $\mathrm{GAE} / \mathrm{ml}$ of extract).

\section{E. GC-MS Analysis of Chemical Components}

The chemical compositions of crude extracts and all 
fractions were analyzed by gas chromatograph (GC-Agilent $6890)$ equipped with HP5-MS $(30 \mathrm{~m} \times 0.25 \mathrm{~mm} \times 0.25 \mu \mathrm{m}$ film thinkness) interfaced to a mass selective detector (HP 5973). The oven temperature was started from $60^{\circ} \mathrm{C}$ to $280{ }^{\circ} \mathrm{C}$ at a rate $3^{\circ} \mathrm{C} / \mathrm{min}$ and held for $16.67 \mathrm{~min}$. Injector temperature was $250{ }^{\circ} \mathrm{C}$. A split ratio of injection was $10: 1$. Helium was used as a carrier gas with the flow of $1 \mathrm{ml} / \mathrm{min}$. The mass spectrometer was operated in electron impact (EI) mode with an electron energy at $70 \mathrm{eV}$. All chemical compounds were identified by matching their mass spectra with reference spectra in the W8N08 and Wiley7n mass spectral libraries (Agilent Technologies, U.S.A.) and compared their retention indices with the published Kováts retention indices, relative to $\mathrm{C} 8-\mathrm{C} 22$ n-alkanes.

\section{F. Statistical Data Analysis}

Significant differences were employed at for $P<0.05$. All analyzes were conducted using variance (ANOVA) test of CropStat version 7.2.3 (CropStat, IRRI).

\section{RESULT AND DISCUSSION}

The Riceberry bran was extracted using solvents (hexane, dichloromethane and methanol) yielding three crude extracts; crude hexane as viscous yellow oil (14.11\%), crude dichloromethane as viscous dark green oil $(4.42 \%)$ and crude methanol as viscous dark purple oil $(5.87 \%)$. These extract were tested for radical scavenging and total phenolic contents and were then subjected to separation by GC-MS in order to identify the chemical constituents. GC-MS chromatogram of fraction CM6 is shown in Fig. 1.

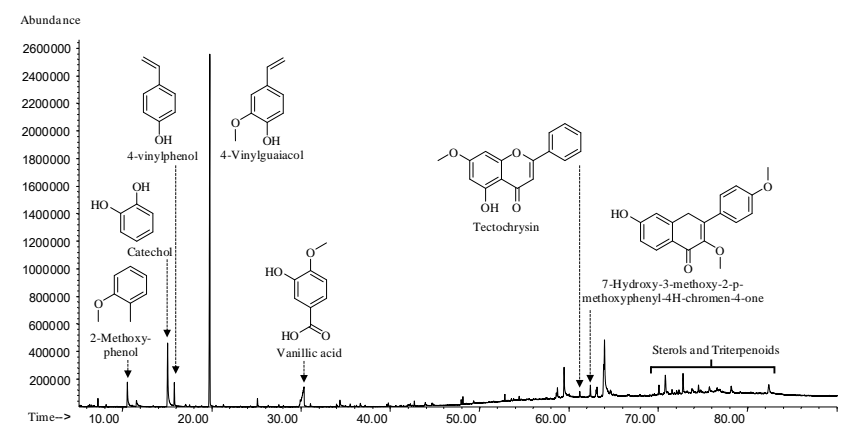

Fig. 1. GC-MS chromatogram of the Fraction CM6. total phenolic contents of the extracts were presented in Table I. Among all the extracts, the crude methanol extract (\% radical scavenger $=94.83 \%$ ) exhibited the highest scavenging ability for DPPH radicals, the crude dichloromethane extract performed rather moderate activity (49.61\%), but the crude hexane did not have an antioxidant activity.

In Folin-Ciocalteu's assay, the standard curve equation for total phenolic content $(\mathrm{mg} \mathrm{GAE} / \mathrm{mL})$ was $\quad y=0.0051 x-$ $0.004\left(R^{2}=0.9993\right)$. Among all, the methanol extract contained the highest amounts of total phenolic $(9.87 \mathrm{mg}$ $\mathrm{GAE} / \mathrm{ml}$ of extract) follow by the dichloromethane extract (3.52 $\mathrm{mg} \mathrm{GAE} / \mathrm{ml}$ of extract). Whereas, crude hexane extract did not show total phenolic content.

TABLE I: Percentage of RAdicAl Scavenging And Total PhenoliC

\begin{tabular}{ccc}
\multicolumn{3}{c}{ CONTENTS OF THE EXTRACTS } \\
\hline Extract & $\begin{array}{c}\text { \% Radical } \\
\text { Scavenger }\end{array}$ & $\begin{array}{c}\text { Total Phenolic } \\
\text { Content }(\mathbf{m g} / \mathbf{m l})\end{array}$ \\
\hline Crude Hexane & $0.00 \pm 0.00^{\mathrm{c}}$ & $0.72 \pm 0.41^{\mathrm{c}}$ \\
Crude Dichloromethane & $49.61 \pm 0.47^{\mathrm{b}}$ & $3.52 \pm 0.00^{\mathrm{b}}$ \\
Crude Methanol & $94.83 \pm 0.08^{\mathrm{a}}$ & $9.87 \pm 0.93^{\mathrm{a}}$ \\
\hline
\end{tabular}

Because the crude methanol extract showed the highest value both of radical scavenging assay and total phenolic content, the extract was considered to be further isolate by column chromatography into 10 fractions with a percentage yield of $20.03,3.93,21.63,4.73,1.93,2.43,1.20,21.17$, 12.10 and $8.49(\mathrm{w} / \mathrm{w})$, respectively. These fractions also were analyzed by GC-MS in order to identify the chemical constituents. Their relative area percentages and their retention indices (RI) are summarized in Table II. Overall, 38 constituents were identified among three crude extracts. However, after the fractionation process, the identified constituents were raised up to 79 compounds. These compounds could be categorized as 3 aliphatic hydrocarbons, 5 aliphatic aldehydes, an aliphatic alcohols, 2 cyclic ketones, 6 aromatics, 8 heterocyclics, 9 simple phenolics, 29 fatty acids and fatty acid esters, 2 diterpenes, 4 flavonoids, 6 sterols, 1 triterpene and 3 cycloartane triterpenoids. The fractionation by column chromatography could enhance the ability to identify chemical components in such complex rice bran extracts.

The percentage of radical scavenger (DPPH assay) and

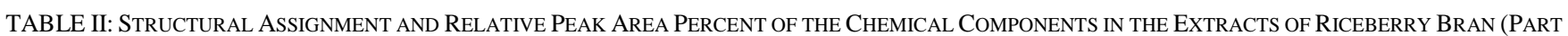
I)

\begin{tabular}{|c|c|c|c|c|c|c|c|}
\hline \multirow{2}{*}{ Structural Assignment $^{\mathrm{a}}$} & \multirow{2}{*}{$\mathbf{R I}$} & \multicolumn{6}{|c|}{ Relative Abundance (\%) } \\
\hline & & CH & CDCM & $\mathbf{C M}$ & CM1 & CM2 & CM3 \\
\hline \multicolumn{8}{|l|}{ Aliphatic Hydrocarbons } \\
\hline 1-Decene ${ }^{1,2}$ & 1100 & & & & & 0.06 & 0.01 \\
\hline Neophytadiene $e^{1,2}$ & 1841 & & & & & & \\
\hline 3,7,11,15-Tetramethyl-2-hexadecene $e^{1,2}$ & 1848 & & & & & & \\
\hline \multicolumn{8}{|l|}{ Aliphatic Aldehydes } \\
\hline Heptenal ${ }^{1,2,3}$ & 965 & 0.20 & & & & & 0.07 \\
\hline (E)-2-Decenal ${ }^{1,2}$ & 1269 & 0.39 & & & & & 0.11 \\
\hline$(\mathrm{E}, \mathrm{Z})-2,4-$ Decadienal ${ }^{1,2}$ & 1301 & & & & & & 0.05 \\
\hline$(\mathrm{E}, \mathrm{E})-2,4-$ Decadienal $^{1,2}$ & 1323 & & & & & & 0.06 \\
\hline \multicolumn{8}{|l|}{ (Z)-9-Octadecenal ${ }^{1}$} \\
\hline \multicolumn{8}{|l|}{ Aliphatic Alcohols } \\
\hline 3,7,11,15-Tetramethyl-2-hexadecen-1-ol ${ }^{1,2}$ & 1883 & & & & & & \\
\hline \multicolumn{8}{|l|}{ Cyclic Ketones } \\
\hline 1,2-Cyclopentanedione $\mathrm{e}^{1,2}$ & 934 & & & 1.53 & & & \\
\hline 4,7,7-Trimethylbicyclo[3,3,0]octan-2-one $\mathrm{e}^{1,2}$ & 1125 & & & & & & \\
\hline
\end{tabular}




\begin{tabular}{|c|c|c|c|c|c|c|c|}
\hline \multicolumn{8}{|l|}{ Aromatics } \\
\hline 1-Ethyl-3-methyl-benzene ${ }^{1,2}$ & 964 & & & & & & \\
\hline $1,2,4$-Trimethyl-benzene $e^{1,2}$ & 972 & & & & & & \\
\hline 1-Ethyl-4-methyl- benzene $e^{1,2}$ & 992 & & & & & & \\
\hline $1,2,3$-Trimethyl-benzene $e^{1,2}$ & 1006 & & & & & & \\
\hline Benzene acitic acid ${ }^{1,2}$ & 1258 & & & & & & \\
\hline 1,4-Dihydrophenanthrene ${ }^{1,2}$ & 1572 & & & & & & \\
\hline \multicolumn{8}{|l|}{ Heteroacyclics } \\
\hline 2,2-Dimethyl-1,3-dioxolane-4-methanol ${ }^{1,2}$ & 947 & & & & & & \\
\hline $2 \mathrm{H}-$ Pyran-2,6(3H)-dione ${ }^{1,2}$ & 1005 & & & 0.12 & & & \\
\hline Thymine $^{1,2}$ & 1086 & & & & & & \\
\hline Methyl nicotinate $e^{1,2}$ & 1146 & & & & & & \\
\hline 2,3-Dihydro-3,5-dihydroxy-6-methyl-4H-pyran-4-one ${ }^{1,2}$ & 1149 & & & 0.26 & & & \\
\hline 5-Hydroxymethyl-2-furancarboxaldehyde $\mathrm{e}^{1,2}$ & 1237 & & & 1.05 & & & \\
\hline Niacin $^{1,2}$ & 1242 & & & & & & \\
\hline 4-(1-Pyrrolyl)butanoic acid ${ }^{1,2}$ & 1374 & & & & & & \\
\hline \multicolumn{8}{|l|}{ Simple Phenolics } \\
\hline 2-Methoxy-phenol (Guaiacol) 1,2 & 1100 & & & & & & \\
\hline 1,2-Benzenediol (Catechol) ${ }^{1,2}$ & 1212 & & & 0.68 & & & \\
\hline 4-Vinylphenol ${ }^{1,2}$ & 1229 & & & & & & \\
\hline $\begin{array}{l}\text { 2-Methoxy-vinylphenol } \\
\text { (4-vinylguaiacol) }\end{array}$ & 1322 & & 0.29 & 0.46 & & & \\
\hline 2,6-Dimethoxy-phenol (Syringol) ${ }^{1,2}$ & 1358 & & & & & & \\
\hline 4-Hydroxy-benzeneethanol (Tyrosol) $)^{1,2}$ & 1431 & & & & & & \\
\hline Methyl 4-hydroxy-3-methoxybenzoate (Methyl vanillate) ${ }^{1,2}$ & 1522 & & & 0.51 & & & 0.03 \\
\hline 4-Hydroxy-3-methoxybenzoic acid(Vanillic acid) ${ }^{1,2}$ & 1571 & & & 0.69 & & & \\
\hline Methyl 3,5-Dihydroxybenzoate ${ }^{1,2}$ & 1688 & & & 0.34 & & & \\
\hline \multicolumn{8}{|l|}{ Fatty Acids and Fatty acid Esters } \\
\hline Octanoic acid ${ }^{1,2}$ & 1184 & & & & & & 0.05 \\
\hline Dodecyl acrylate ${ }^{1,2}$ & 1697 & & & & & & \\
\hline 2-(Dodecyloxy)-ethanol ${ }^{1,2}$ & 1719 & & & & & 0.10 & \\
\hline Methyl myristate ${ }^{1,2}$ & 1729 & & 0.03 & 0.27 & & 0.27 & 0.03 \\
\hline Myristic acid ${ }^{1,2}$ & 1768 & 0.18 & 0.26 & 0.17 & & & 0.01 \\
\hline Methyl 9-methyltetradecanoate ${ }^{1,2}$ & 1828 & & & & & 0.03 & \\
\hline (Z)-Methyl 9-hexadecenoate ${ }^{1,2}$ & 1905 & & & & & 0.13 & \\
\hline Methyl palmitate ${ }^{1,2}$ & 1929 & & 1.49 & 7.35 & & 13.58 & 1.84 \\
\hline Palmitic Acid ${ }^{1,2,3}$ & 1974 & 12.95 & 13.6 & 9.72 & & 1.83 & 9.31 \\
\hline Ethyl palmitate $^{1,2}$ & 1995 & & 0.03 & & & 0.14 & \\
\hline Methyl 8,11-octadecadienoate ${ }^{1,2}$ & 2094 & & 3.12 & 8.66 & & 17.48 & 2.47 \\
\hline Methyl oleate $e^{1,2}$ & 2101 & & 4.12 & 10.65 & & 27.59 & 3.45 \\
\hline Methyl stearate ${ }^{1,2}$ & 2127 & & & 0.07 & & 1.93 & \\
\hline Linoleic acid $^{1,2}$ & 2131 & 76.91 & 62.3 & 18.83 & & & \\
\hline Oleic acid $^{1,2,3}$ & 2156 & & & 14.55 & & 9.93 & 64.66 \\
\hline Ethyl linoleate $^{1,2}$ & 2160 & & & & & 0.42 & \\
\hline Ethyl Oleate ${ }^{1,2}$ & 2167 & & & & & 0.78 & \\
\hline Stearic acid ${ }^{1,2}$ & 2172 & & & 0.70 & & & \\
\hline Methyl 11-eicosenoate $e^{1,2}$ & 2294 & & & 0.13 & & 0.49 & \\
\hline Methyl arachidate ${ }^{1}$ & & & & 0.04 & & 0.80 & 0.09 \\
\hline Arachidic acid $^{1}$ & & & & 0.16 & & & \\
\hline \multicolumn{8}{|l|}{ Butyl 9,12-octadecadienoate ${ }^{1}$} \\
\hline 2-Hydroxy-1-(hydroxypropyl) ethyl hexadecanoate ${ }^{1}$ & & & & 0.90 & & & \\
\hline Methyl behenate $^{1}$ & & & & 0.90 & & 0.25 & \\
\hline \multicolumn{8}{|l|}{ 3-Hydroxypropyl oleate ${ }^{1}$} \\
\hline$\beta$-Monolinolein ${ }^{1}$ & & & & 0.91 & & & \\
\hline 3-Hydroxypropyl oleate ${ }^{1}$ & & & & 1.98 & & & \\
\hline Methyl tetracosanoate ${ }^{1}$ & & & & 0.14 & & & \\
\hline Methyl hexacosanoate ${ }^{1}$ & & & & & & 0.11 & 0.03 \\
\hline \multicolumn{8}{|l|}{ Diterpenes } \\
\hline Phytol $^{1}$ & 2111 & & & & & & \\
\hline $\begin{array}{l}\text { 2-Methyl hydrogen } \\
\text { 8,13-epoxy-3-oxo-1,2-secolabdane-1,2-dioat }{ }^{1}\end{array}$ & & & & & 17.51 & 1.80 & 0.22 \\
\hline \multicolumn{8}{|l|}{ Flavonoids } \\
\hline Tectochrysin ${ }^{1}$ & & & & & & & 0.14 \\
\hline 7-Hydroxy-3-methoxy-2-p-methoxyphenyl-4H-chromen-4-one ${ }^{1}$ & & & & & & & 0.14 \\
\hline Apigenin $^{1}$ & & & & & & & \\
\hline $\begin{array}{l}\text { 2-(3-Hydroxy-4-methoxyphenyl)-3,7-dimethoxy-4H-chromen-4 } \\
\text {-one }{ }^{1}\end{array}$ & & & & & & & 0.12 \\
\hline \multicolumn{8}{|l|}{ Sterols } \\
\hline Stigmasta-3,5,22-trien $^{1}$ & & & & & 3.26 & 0.34 & 0.03 \\
\hline Ergosta-4,6,22-trien-3 $\beta$-ol ${ }^{1}$ & & & 0.4 & 0.4 & & 0.27 & \\
\hline Stigmastan-3,5-diene ${ }^{1}$ & & & & & 12.43 & 1.46 & 0.17 \\
\hline
\end{tabular}




\begin{tabular}{|c|c|c|c|c|c|c|}
\hline Campestrol $^{1}$ & 0.73 & 0.58 & 0.38 & \multirow[t]{2}{*}{3.10} & & 0.12 \\
\hline Stigmasterol $^{1,3}$ & 0.50 & 0.56 & 0.58 & & & 0.11 \\
\hline$\beta$-Sitosterol ${ }^{1,3}$ & 1.58 & 1.61 & 1.24 & 2.49 & & 0.22 \\
\hline \multicolumn{7}{|l|}{ Triterpene } \\
\hline Squalene ${ }^{1}$ & & 0.29 & 0.23 & 23.50 & & \\
\hline \multicolumn{7}{|l|}{ Cycloartane Triterpenoids } \\
\hline Cycloartenol $^{1}$ & & & & & & 0.17 \\
\hline 24-Methylenecycloartan-3-one & & 1.37 & & & 1.64 & 0.35 \\
\hline 24-Methylene-cycloartanol ${ }^{1}$ & 0.64 & & 0.64 & 7.02 & 0.29 & 1.26 \\
\hline
\end{tabular}

TABLE II: Structural Assignment and Relative Peak ARea Percent of The Chemical Components in the Extracts of RiceberRy BRan (Part II)

\begin{tabular}{|c|c|c|c|c|c|c|c|}
\hline \multirow{2}{*}{ Structural Assignment $^{\mathrm{a}}$} & \multicolumn{7}{|c|}{ Relative Abundance (\%) } \\
\hline & CM4 & CM5 & CM6 & CM7 & CM8 & CM9 & CM10 \\
\hline \multicolumn{8}{|l|}{ Aliphatic Hydrocarbons } \\
\hline 1 -Decene $e^{1,2}$ & 0.52 & 0.69 & & & 0.50 & & \\
\hline Neophytadiene $^{1,2}$ & 0.80 & & & & & & \\
\hline $3,7,11,15$-Tetramethyl-2-hexadecene ${ }^{1,2}$ & 0.17 & & & & & & \\
\hline \multicolumn{8}{|l|}{ Aliphatic Aldehydes } \\
\hline Heptenal ${ }^{1,2,3}$ & 0.71 & & 0.09 & & & & \\
\hline (E)-2-Decenal ${ }^{1,2}$ & 0.66 & & & & & & \\
\hline$(\mathrm{E}, \mathrm{Z})-2,4$-Decadienal ${ }^{1,2}$ & 0.35 & & & & & & \\
\hline$(\mathrm{E}, \mathrm{E})-2,4$-Decadienal ${ }^{1,2}$ & 0.46 & & & & & & \\
\hline (Z)-9-Octadecenal ${ }^{1}$ & 2.45 & & & & & & \\
\hline \multicolumn{8}{|l|}{ Aliphatic Alcohols } \\
\hline $3,7,11,15$-Tetramethyl-2-hexadecen-1-ol ${ }^{1,2}$ & 0.17 & & & & & & \\
\hline \multicolumn{8}{|l|}{ Cyclic Ketones } \\
\hline 1,2 -Cyclopentanedione $\mathrm{e}^{1,2}$ & & & & 4.22 & 3.27 & 0.58 & 10.06 \\
\hline 4,7,7-Trimethylbicyclo $[3,3,0]$ octan-2-one ${ }^{1,2}$ & & & 0.90 & & & & \\
\hline \multicolumn{8}{|l|}{ Aromatics } \\
\hline 1-Ethyl-3-methyl-benzene $e^{1,2}$ & & & 0.19 & & & & \\
\hline $1,2,4$-Trimethyl-benzene ${ }^{1,2}$ & & & 0.10 & 0.14 & & & \\
\hline 1-Ethyl-4-methyl- benzene ${ }^{1,2}$ & & & 0.06 & & & & \\
\hline $1,2,3$-Trimethyl-benzene ${ }^{1,2}$ & & & & 1.49 & & & \\
\hline Benzene acitic acid ${ }^{1,2}$ & & & 0.90 & & & & \\
\hline 1,4-Dihydrophenanthrene $e^{1,2}$ & & & & & & 0.65 & \\
\hline \multicolumn{8}{|l|}{ Heteroacyclics } \\
\hline 2,2-Dimethyl-1,3-dioxolane-4-methanol ${ }^{1,2}$ & & & & 1.41 & & & \\
\hline $2 \mathrm{H}-P y r a n-2,6(3 \mathrm{H})$-dione ${ }^{1,2}$ & & & & & & & 1.58 \\
\hline Thymine $^{1,2}$ & & & & & & 2.44 & 0.52 \\
\hline Methyl nicotinate ${ }^{1,2}$ & & & & & & 0.35 & 1.17 \\
\hline 2,3-Dihydro-3,5-dihydroxy-6-methyl-4H-pyran-4-one ${ }^{1,2}$ & & & & & & & 0.34 \\
\hline 5-Hydroxymethyl-2-furancarboxaldehyde ${ }^{1,2}$ & & & & & & 0.34 & \\
\hline $\mathrm{Niacin}^{1,2}$ & & & & & & 0.60 & 0.34 \\
\hline 4-(1-Pyrrolyl)butanoic acid ${ }^{1,2}$ & & & & & & & 0.53 \\
\hline \multicolumn{8}{|l|}{ Simple Phenolics } \\
\hline 2-Methoxy-phenol (Guaiacol ) $)^{1,2}$ & & & 3.37 & & & 0.68 & 0.67 \\
\hline 1,2-Benzenediol (Catechol) ${ }^{1,2}$ & & & 8.71 & 11.62 & 1.77 & 1.88 & 0.95 \\
\hline 4-Vinylphenol ${ }^{1,2}$ & & & 1.96 & 0.51 & & & \\
\hline $\begin{array}{l}\text { 2-Methoxy-vinylphenol } \\
\text { (4-vinylguaiacol) } \text { (1,2 }^{2}\end{array}$ & & & 35.00 & 10.44 & 1.30 & 0.72 & 0.57 \\
\hline 2,6-Dimethoxy-phenol (Syringol) 1,2 & & & & 0.52 & & & \\
\hline 4-Hydroxy-benzeneethanol (Tyrosol) $)^{1,2}$ & & & 0.09 & & & & \\
\hline Methyl 4-hydroxy-3-methoxybenzoate (Methyl vanillate) ${ }^{1,2}$ & 0.31 & & & 0.22 & 0.40 & 0.35 & 0.50 \\
\hline 4-Hydroxy-3-methoxybenzoic acid(Vanillic acid) ${ }^{1,2}$ & & & 0.99 & 2.52 & 1.93 & 0.85 & 0.39 \\
\hline Methyl 3,5-Dihydroxybenzoate ${ }^{1,2}$ & & & 0.73 & & & & \\
\hline \multicolumn{8}{|l|}{ Fatty Acids and Fatty acid Esters } \\
\hline Octanoic acid ${ }^{1,2}$ & 0.23 & & & & & & \\
\hline Dodecyl acrylate ${ }^{1,2}$ & 2.18 & & & & & & \\
\hline 2-(Dodecyloxy)-ethanol ${ }^{1,2}$ & & 1.00 & & & 0.88 & & \\
\hline \multicolumn{8}{|l|}{ Methyl myristate ${ }^{1,2}$} \\
\hline \multicolumn{8}{|l|}{ Myristic acid ${ }^{1,2}$} \\
\hline \multicolumn{8}{|l|}{ Methyl 9-methyltetradecanoate ${ }^{1,2}$} \\
\hline \multicolumn{8}{|l|}{ (Z)-Methyl 9-hexadecenoate ${ }^{1,2}$} \\
\hline Methyl palmitate ${ }^{1,2}$ & 2.26 & 1.02 & 0.42 & & 0.14 & & \\
\hline Palmitic Acid ${ }^{1,2,3}$ & & 0.68 & 0.40 & 1.23 & 8.29 & & 6.44 \\
\hline \multicolumn{8}{|l|}{ Ethyl palmitate ${ }^{1,2}$} \\
\hline Methyl 8,11-octadecadienoate ${ }^{1,2}$ & 4.44 & 1.32 & 0.42 & & & 0.35 & 12.84 \\
\hline Methyl oleate ${ }^{1,2}$ & 4.51 & 1.87 & & & & & \\
\hline Methyl stearate ${ }^{1,2}$ & 0.31 & & & & & & \\
\hline
\end{tabular}




\begin{tabular}{|c|c|c|c|c|c|c|c|}
\hline Linoleic acid ${ }^{1,2}$ & & & & & 2.21 & 2.00 & \\
\hline Oleic acid ${ }^{1,2,3}$ & & & & & 16.36 & 1.16 & \\
\hline \multicolumn{8}{|l|}{ Ethyl linoleate ${ }^{1,2}$} \\
\hline \multicolumn{8}{|l|}{ Ethyl Oleate ${ }^{1,2}$} \\
\hline \multicolumn{8}{|l|}{ Stearic acid ${ }^{1,2}$} \\
\hline \multicolumn{8}{|l|}{ Methyl 11-eicosenoate ${ }^{1,2}$} \\
\hline \multicolumn{8}{|l|}{ Methyl arachidate ${ }^{1}$} \\
\hline \multicolumn{8}{|l|}{ Arachidic acid ${ }^{1}$} \\
\hline Butyl 9,12-octadecadienoate ${ }^{1}$ & 1.02 & & & & & & \\
\hline 2-Hydroxy-1-(hydroxypropyl) ethyl hexadecanoate ${ }^{1}$ & & & 3.78 & & & 1.07 & \\
\hline \multicolumn{8}{|l|}{ Methyl behenate ${ }^{1}$} \\
\hline 3-Hydroxypropyl oleate ${ }^{1}$ & & & & & 3.94 & 4.52 & \\
\hline \multicolumn{8}{|l|}{$\beta$-Monolinolein ${ }^{1}$} \\
\hline \multicolumn{8}{|l|}{ 3-Hydroxypropyl oleate ${ }^{1}$} \\
\hline \multicolumn{8}{|l|}{ Methyl tetracosanoate ${ }^{1}$} \\
\hline \multicolumn{8}{|l|}{ Methyl hexacosanoate $^{1}$} \\
\hline \multicolumn{8}{|l|}{ Diterpenes } \\
\hline Phytol $^{1}$ & 0.26 & & & & & & \\
\hline 2-Methyl hydrogen 8,13-epoxy-3-oxo-1,2-secolabdane-1,2-dioat ${ }^{1}$ & 4.38 & & & 11.35 & 7.31 & 5.02 & 4.09 \\
\hline \multicolumn{8}{|l|}{ Flavonoids } \\
\hline Tectochrysin ${ }^{1}$ & & & 0.60 & 1.33 & & & \\
\hline 7-Hydroxy-3-methoxy-2-p-methoxyphenyl-4H-chromen-4-one ${ }^{1}$ & & & 1.00 & 2.14 & & & \\
\hline Apigenin $^{1}$ & & & & & & 5.97 & \\
\hline 2-(3-Hydroxy-4-methoxyphenyl)-3,7-dimethoxy-4H-chromen-4-one & & & & 4.02 & & & \\
\hline \multicolumn{8}{|l|}{ 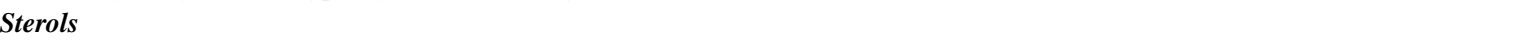 } \\
\hline Stigmasta-3,5,22-trien ${ }^{1}$ & 0.90 & 3.37 & 0.54 & 2.08 & 0.73 & 1.20 & \\
\hline \multicolumn{8}{|l|}{ Ergosta-4,6,22-trien-3 $\beta$-ol ${ }^{1}$} \\
\hline Stigmastan-3,5-diene ${ }^{1}$ & 3.42 & 13.63 & 2.04 & & 4.84 & 3.63 & 3.62 \\
\hline Campestrol $^{1}$ & 10.85 & 2.13 & 1.13 & 2.00 & 1.65 & 1.18 & 0.87 \\
\hline Stigmasterol $^{1,3}$ & 10.27 & 1.20 & & & & & \\
\hline$\beta$-Sitosterol ${ }^{1,3}$ & 21.25 & 7.15 & 0.95 & 1.97 & 1.25 & 0.77 & 1.18 \\
\hline \multicolumn{8}{|l|}{ Triterpene } \\
\hline \multicolumn{8}{|l|}{ Squalene $^{1}$} \\
\hline \multicolumn{8}{|l|}{ Cycloartane Triterpenoids } \\
\hline \multicolumn{8}{|l|}{ Cycloartenol $^{1}$} \\
\hline \multicolumn{8}{|l|}{ 24-Methylenecycloartan-3-one ${ }^{1}$} \\
\hline 24-Methylene-cycloartanol ${ }^{1}$ & 8.77 & 4.79 & 1.47 & 3.91 & 2.60 & 2.67 & 1.58 \\
\hline
\end{tabular}

${ }^{a}$ Identification: 1, mass spectrum (tentative); 2, retention indices (RI); and 3, standard compound.

The major component in all of the three crude extracts was linoleic acid $(76.91 \%, 62.3 \%$ and $18.83 \%)$ and the major compounds of the methanol fractionates were squalene $(23.50 \%)$, methyl oleate $(27.59 \%)$, oleic acid $(64.66 \%)$, $\beta$-sitosterol $\quad(21.25 \%), \quad$ stigmastan-3,5-diene(13.63\%), 4-vinylguaiacol (35.00\%), catechol (11.62\%), oleic acid $(16.36 \%)$ apigenin $(5.97 \%)$ and methyl 8,11 -octadecadienoate $(12.84 \%)$, respectively.

Among these identified compounds, simple phenolics and flavonoids are responsible for antioxidantion, anti-inflammation and anticancer, for instance, 4-vinylguaiacol is a flavoring substance with an antioxidant property [8], apigenin has antioxidant, anti-inflammatory and anticancer activities [9].

Simple phenolics were reported to possess important biological activities and to be especially involved in the reduction of oxidative stress as a major cause of age-related diseases and cancers [10].The identified simple phenolics in the rice bran extracts were guaiacol, catechol, 4-vinylphenol, 4-vinylguaiacol, syringol, tyrosol, methyl vanillate, vanillic acid and methyl 3,5-dihydroxybenzoate.

Flavonoids are typical phenolic compounds and powerful chain-breaking antioxidants. The identified flavonoids in the extracts were tectochrysin, 7-hydroxy-3-methoxy-2-pmethoxyphenyl-4H-chromen-4-one, apigenin and 2-(3hydroxy-4-methoxyphenyl)-3,7-dimethoxy-4H-chromen-4one.
It is implied that simple phenolics and flavonoids present in the methanol extract of Riceberry bran play an important role in antioxidant activity.

\section{CONCLUSION}

Identification of some antioxidants in bran of the Thai black rice cultivar Riceberry was accomplished using solvent extraction, fractionation by column chromatography, and then analysis by GC-MS. All crude extracts were also investigated for antioxidant capacity and total phenolic content. The study revealed that the crude methanolic extract of Riceberry bran had the strongest antioxidant capacity. Among overall identified compounds, simple phenolic compounds and flavonoids were antioxidant compounds. The major phenolic compounds in this rice bran was 4-vinylguaiacol and the major flavonoids in which was apigenin. The result showed that Riceberry rice had great potential for use as a functional food.

\section{ACKNOWLEDGMENT}

This study was funded by the Nation Research Council of Thailand (NRCT) and a grant under the Strategic Scholarships for Frontier Research Network for the Ph.D. Program Thai Doctoral degree from the Commission on Higher Education (CHE), Thailand was given to $\mathrm{P}$. 
Suttiarporn. We thank the Center of Excellence for Innovation in Chemistry (PERCH-CIC), and the Graduate School, Chiang Mai University for their partial supports, and Dr. Apichart Vanavichit of the Rice Science Center and Rice Gene Discovery Unit, Kasetsart University, Kamphangsaen Campus for providing the Riceberry black rice.

\section{REFERENCES}

[1] K. Pitija, M. Nakornriab, T. Sriseadka, A. Vanavichit, and S Wongpornchai, "Anthocyanin content and antioxidant capacity in bran extracts of some Thai black rice varieties," Int. J. Food Sci. Tech., vol. 48, no. 2, pp. 300-308, 2013.

[2] T. Sriseadka, S. Wongpornchai, and M. Rayanakorn, "Quantification of flavonoids in black rice by liquid chromatography-negative electrospray ionization tandem mass spectrometry," J. Agric. Food Chem., vol. 60, no. 47, pp. 11723-11732, 2012.

[3] S. Yodpitak, P. Sookwong, P. Akkaravessapong, and S. Wongpornchai, "Changes in antioxidant activity and antioxidative compounds of brown rice after pre-germination," J. Food Nutr. Res., vol. 1, no. 6, pp. 132-137, 2012.

[4] P. Sookwong, K. Nakagawa, K. Murata, Y. Kojima, and T. Miyazawa, "Quantitation of tocotrienol and tocopherol in various rice brans," $J$. Agric. Food Chem., vol. 55, no. 2, pp. 461-466, 2012.

[5] G. Pereira-Caro, S. Watanabe, A. Crozier, T. Fujimura, T. Yokota, and H. Ashihara, "Phytochemical profile of a Japanese black-purple rice," Food Chem., vol. 141, no. 3, pp. 2821-2827. 2012.

[6] V. Leardkamolkarn, W. Thongthep, P. Suttiarporn, R. Kongkachuichai, S. Wongpornchai, and A. Wanavijitr, "Chemopreventive properties of the bran extracted from a newly-developed Thai rice: The Riceberry," Food Chem., vol. 125, no. 3, pp. 978-985, 2011.

[7] P. Prangthip, R. Surasiang, R. Charoensiri, V. Leardkamolkarn, S. Komindr, U. Yamborisut, A. Vanavichit, and R. Kongkachuichai, "Amelioration of hyperglycemia, hyperlipidemia, oxidative stress and inflammation in steptozotocin-induced diabetic rats fed a high fat diet by riceberry supplement," J. Funct. Foods, vol. 5, no. 1, pp. 195-203, 2013.

[8] J. B. Jeong and H. J. Jeong, "2-Methoxy-4-vinylphenol can induce cell cycle arrest by blocking the hyper-phosphorylation of retinoblastoma protein in benzo[a]pyrene-treated NIH3T3 cells," Biochem. Biophys. Res. Commun., vol. 400, no. 4, pp. 752-757, 2010.

[9] D. Patel, S. Shukla, and S. Gupta, "Apigenin and cancer chemoprevention: Progress, potential and promise (review)," Int. J. Oncol., vol. 30, pp. 233-245, 2007.

[10] M. K. Pyo, J. L. Jin , Y. K. Koo, and H. S.Yun-Choi, "Phenolic and furan type compounds isolated from Gastrodia elata and their anti-platelet effects," Arch. Pharm. Res., vol. 27, no. 4, pp. 381-385, 2004.

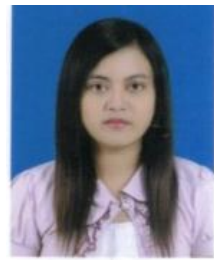

Panawan Suttiarporn is a Ph.D. student in organic chemistry, the Department of Chemistry, Faculty of Science, Chiang Mai University, Thailand. She got her B.Sc. degree in chemistry in 2006 from Chiang Mai University, Thailand. Her current research interests include chromatographic-mass spectrometric technique for analysis of phytochemicals in rice and natural products.

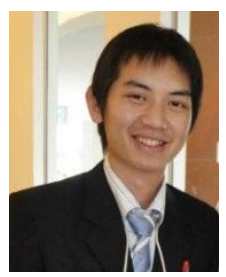

Phumon Sookwong received his doctoral degree in agricultural science from Tohoku University, Japan, in 2009 . He is specialized in researching the analysis and development of food and nutraceuticals.

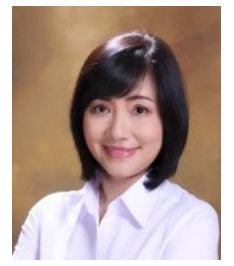

Sugunya Mahatheeranont received her doctora degree from University of Wales Cardiff, in United Kingdom. She is specialized in researching the rice phytochemicals by combined chromatographic-mass spectrometric, and hyphenated techniquese; GC-MS, GCxGC-MS, HPLC-MS/MS. 